\title{
Cream plus Soft Vaginal Capsule Dosage Form
}

National Cancer Institute

\section{Source}

National Cancer Institute. Cream plus Soft Vaginal Capsule Dosage Form. NCI

Thesaurus. Code C149411.

Combination package consisting of a cream and a soft vaginal capsule. 